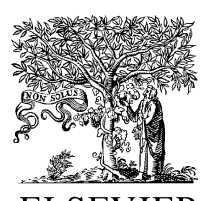

ELSEVIER

\title{
Numerical modelling of turbulent catalytically stabilized channel flow combustion
}

\author{
John Mantzaras*, Christoph Appel, Peter Benz, Urs Dogwiler \\ Paul Scherrer Institute, Combustion Research, CH-5232 Villigen PSI, Switzerland
}

\begin{abstract}
The turbulent catalytically stabilized combustion of lean hydrogen-air premixtures is investigated numerically in plane channels with platinum-coated isothermal walls. The catalytic wall temperature is $1220 \mathrm{~K}$ and the incoming mixture has a mean velocity of $15 \mathrm{~m} / \mathrm{s}$ and a turbulent kinetic energy of $1.5 \mathrm{~m}^{2} / \mathrm{s}^{2}$. A two-dimensional elliptic model is developed with elementary heterogeneous and homogeneous chemical reactions. The approach is based on a two-layer $k-\varepsilon$ model of turbulence, a Favre-average moment closure, a presumed-shape (Gaussian) probability density function for gaseous reactions, and a laminar-like closure for surface reactions. Gaseous combustion is confined close to the catalyst surface due to the diffusional imbalance of the limiting reactant (hydrogen). In addition, the peak rms temperature and species fluctuations are always located outside the extent of the homogeneous reaction zone indicating that thermochemical fluctuations have no significant influence on gaseous combustion. Turbulence is significantly suppressed by gaseous combustion resulting in higher turbulent transport for the leaner mixtures, a successive push of the gaseous reaction zone towards the wall, incomplete combustion, and subsequent catalytic conversion of the leaked fuel. Comparison between turbulent and laminar cases having the same incoming properties shows that turbulence inhibits homogeneous ignition due to increased heat transport away from the near-wall layer. (C) 2000 Elsevier Science B.V. All rights reserved.
\end{abstract}

Keywords: Two-dimensional turbulent combustion modelling; Elementary heterogeneous; Homogeneous chemical reactions

\section{Introduction}

The combustion of fuel-lean gaseous mixtures with very low emissions and enhanced flame stability is of great importance in many practical devices such as gas-fired turbines and burners. In catalytically stabilized combustion (CST) partial fuel conversion is attained heterogeneously in burners with a suitably large surface-to-volume ratio, such as Pt- or Pd-coated honeycomb monoliths. Complete fuel conversion is usually achieved with subsequent homogeneous (gaseous) combustion in a post-catalyst staged gaseous burner [1]. The reduction in $\mathrm{NO}_{x}$ emissions can be then sig-

\footnotetext{
* Corresponding author. Tel.: +56-310-4046; fax: +56-310-2199. E-mail address: mantzaras@psi.ch (J. Mantzaras)
}

nificant, as $\mathrm{NO}_{x}$ is produced solely from the gaseous reaction path $[2,3]$. The onset of homogeneous combustion within the catalytic burner is usually detrimental to the catalyst integrity and this problem is further accentuated by the current trend to use higher temperature catalysts. The coupling between heterogeneous and homogeneous chemistries which is responsible for the onset of gaseous combustion in catalytic burners is hence one important aspect in CST research.

The interactions between heterogeneous and homogeneous chemical reactions leading to homogeneous ignition have been investigated numerically in three basic flow configurations: one-dimensional stagnation point flows, two-dimensional external boundary layer flows, and two-dimensional internal (channel) flows. Stagnation point flows have provided a particularly 
amenable platform for such studies (see, e.g. [4-6]). The regimes of homogeneous hydrocarbon combustion were identified in Song et al. [4] using bifurcation theory and one-step chemistry for both the gaseous and surface kinetics. Ikeda et al. [5] investigated the homogeneous ignition of hydrogen-air mixtures using detailed gaseous and simplified surface kinetics while Bui et al. [6] employed detailed gaseous and surface reaction schemes. The two-dimensional external flow studies included boundary layer (parabolic) models for flows over flat catalytic plates with simplified surface and detailed gaseous chemistries and addressed primarily the effect of radical adsorption-desorption reactions on gaseous ignition [7-10]. Channel flow configurations are the most relevant for practical applications as the catalytic burners are usually of the honeycomb type consisting of a multitude of individual channels. Channel CST has been investigated with elliptic models and simplified surface chemistry with main interest to study the influence of the flow and chemistry parameters on gaseous combustion [11-13]. Elliptic models are better suited to study the foregoing processes since the volumetric gaseous expansion can invalidate the boundary layer approximation. Recently [14] we investigated numerically the homogeneous ignition of lean methane-air mixtures in channel CST using a two-dimensional elliptic model with detailed gaseous and surface chemistries; the key surface reactions affecting homogeneous ignition were identified. Homogeneous ignition distances predicted with this model were then successfully compared to measurements [15], thus strengthening the confidence on the CST applicability of newly developed surface reaction schemes [16].

The flow field in the previous studies was laminar. In many practical devices, however, the incoming flow can be fully turbulent. This is the case, e.g., in gas-fired turbines where following compression the air mixture has developed an extended inertial subrange. The spectral energy content of the incoming turbulence and the cross-sectional area of the catalytic channel determine the active part of the turbulent spectrum entering the channel; spectral energy considerations [17] have shown a strong influence of the small turbulent scales in premixed combustion. Surface and gaseous reactions interact with turbulence and with each other. Turbulence enhances the heat and mass transport coefficients towards-or-away from the catalyst surface and in addition it can be strongly coupled to gaseous combustion due to the increased transport and the induced thermochemical fluctuations. These processes are further complicated by the fact that they occur near a solid surface; near-wall turbulence is not well understood even in simpler non-reacting constant property flows [18]. This study undertakes a first investigation of turbulent CST combustion in plane channel flows. A full elliptic two-dimensional model is developed that includes detailed gas-phase and surface chemistries. The treatment of detailed surface chemistry is crucial given the significance of radical adsorption-desorption reactions on gaseous ignition $[14,15]$. The approach is based on a two-layer $k-\varepsilon$ turbulence model and Favre-averaged modelled transport equations for all second order moments. A presumed Gaussian shape joint probability density function (p.d.f.) is used as a coupling submodel to evaluate the average gaseous reaction rates. The mean heterogeneous reaction rates are modelled as "laminar" rates evaluated at their corresponding mean properties since the thermal inertia of the catalyst support diminishes the surface temperature fluctuations and thus removes the major non-linearity in the surface chemical source terms.

The main objective of this investigation is to examine the influence of fluid mechanical turbulence on CST performance. The effect of turbulence on both heterogeneous and homogeneous combustion is addressed by carrying out a comparative study of lean hydrogen-air CST at different equivalence ratios. This paper is organized as follows. First the channel geometry is presented, the mathematical model along with the numerical procedure follows, and then results are presented for three different cases with-or-without the presence of gaseous reactions. The effect of turbulent fluctuations and turbulent transport on heterogeneous and homogeneous combustion is then discussed. Comparisons are finally made with corresponding laminar cases in order to assess fundamental CST performance differences between the laminar and turbulent flow modes.

\section{Channel geometry and flow conditions}

The two-dimensional channel geometry of the present simulations is illustrated in Fig. 1. It consists of two catalytically active parallel plates with a length 


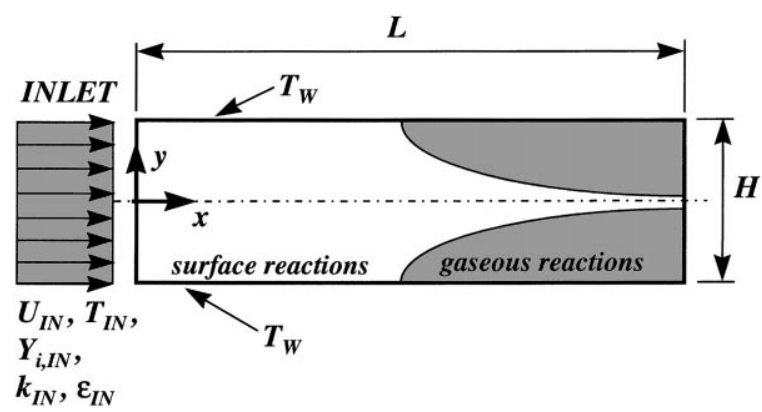

Fig. 1. Schematic of the two-dimensional plane channel flow configuration.

$(L)$ of $150 \mathrm{~mm}$ and a vertical separation $(H)$ of $10 \mathrm{~mm}$. This geometry simulates our optically accessible channel catalytic combustors such as the ones used in [15] and [11]. Preheated fuel-lean hydrogen/air premixtures enter the channel with the following uniform properties: streamwise velocity $U_{\mathrm{IN}}=15 \mathrm{~m} / \mathrm{s}$, temperature $T_{\mathrm{IN}}=400 \mathrm{~K}$, turbulent kinetic energy $k_{\mathrm{IN}}=0.0068 U_{\mathrm{IN}}^{2}$, and dissipation rate of turbulent kinetic energy $\varepsilon_{\mathrm{IN}}=k_{\mathrm{IN}}^{3 / 2} / b$ with $b=0.1 H$. The pressure was atmospheric and the Reynolds number based on the incoming mean properties and the hydraulic diameter of the channel $(2 \mathrm{H})$ was about 11500. The channel walls were considered to be coated with platinum and their temperature was fixed to $1220 \mathrm{~K}$. The catalyst site density $(G)$ was taken $2.7 \times 10^{-8} \mathrm{kmol} / \mathrm{m}^{2}$ simulating polycrystalline platinum as in [19]. The fuel-to-air equivalence ratio was varied between 0.215 and 0.240 . It must be stated that in practical applications higher equivalence ratios are detrimental to the catalyst integrity. This is because $\mathrm{H}_{2}$ is a strongly diffusionally imbalanced fuel with a Lewis number of about 0.3 , resulting in a surface equivalence ratio nearly twice the corresponding one of the gas-phase [6].

\section{Numerical model}

\subsection{Gas-phase modelling}

The present work is based on the numerical solution of the gas-phase transport equations in their Cartesian two-dimensional elliptic form. A moment closure approach is adopted by constructing Favre-averaged modelled transport equations for all gas-phase variables. The transport equation for any variable $\varphi$ is written as (all variables are defined in the Nomenclature)

$$
\begin{gathered}
\frac{\partial(\bar{\rho} \tilde{u} \tilde{\varphi})}{\partial x}+\frac{\partial(\bar{\rho} \tilde{v} \tilde{\varphi})}{\partial y}-\frac{\partial}{\partial x}\left(\Gamma_{\text {eff }} \frac{\partial \tilde{\varphi}}{\partial x}\right) \\
-\frac{\partial}{\partial y}\left(\Gamma_{\text {eff }} \frac{\partial \tilde{\varphi}}{\partial y}\right)=\tilde{S}_{\varphi}
\end{gathered}
$$

with

$\Gamma_{\text {eff }}=\Gamma+\frac{\mu_{\mathrm{t}}}{\sigma_{\varphi}}$.

The equations solved in this study (Eqs. (3)-(12)) are summarized below.

Summary of governing equations: ${ }^{1}$

1. Continuity: ${ }^{2}$

$$
\varphi=1, \quad \tilde{S}_{\varphi}=0 .
$$

2. $x$-momentum:

$$
\begin{aligned}
\varphi= & \tilde{u}, \quad \tilde{S}_{\varphi}=-\frac{\partial \bar{p}}{\partial x}+\frac{\partial}{\partial x}\left(\Gamma_{\text {eff }} \frac{\partial \tilde{u}}{\partial x}\right) \\
& +\frac{\partial}{\partial y}\left(\Gamma_{\text {eff }} \frac{\partial \tilde{v}}{\partial x}\right)-\frac{\partial}{\partial x}\left[\frac{2}{3} \Gamma_{\text {eff }}\left(\frac{\partial \tilde{u}}{\partial x}+\frac{\partial \tilde{v}}{\partial y}\right)\right] \\
& -\frac{\partial}{\partial x}\left(\frac{2}{3} \bar{\rho} \tilde{k}\right) .
\end{aligned}
$$

3. $y$-momentum:

$$
\begin{aligned}
\varphi= & \tilde{v}, \quad \tilde{S}_{\varphi}=-\frac{\partial \bar{p}}{\partial y}+\frac{\partial}{\partial x}\left(\Gamma_{\text {eff }} \frac{\partial \tilde{u}}{\partial y}\right) \\
& +\frac{\partial}{\partial y}\left(\Gamma_{\text {eff }} \frac{\partial \tilde{v}}{\partial y}\right)-\frac{\partial}{\partial y}\left[\frac{2}{3} \Gamma_{\text {eff }}\left(\frac{\partial \tilde{u}}{\partial x}+\frac{\partial \tilde{v}}{\partial y}\right)\right] \\
& -\frac{\partial}{\partial y}\left(\frac{2}{3} \bar{\rho} \tilde{k}\right) .
\end{aligned}
$$

4. Turbulent kinetic energy: ${ }^{3}$

$$
\varphi=\tilde{k}, \quad \tilde{S}_{\varphi}=P_{k}+G_{k}-\bar{\rho} \tilde{\varepsilon} .
$$

\footnotetext{
1 Turbulence constants given in Eqs. (3)-(12) are $C_{\mu}=0.09$, $\mathrm{C}_{1}=1.44, \mathrm{C}_{2}=1.0, \mathrm{C}_{3}=1.92, \sigma_{k}=1.0, \sigma_{\varepsilon}=1.3, \sigma_{\rho}=1.3, \sigma_{g}=0.86$, $C_{g, 1}=2.8, C_{g, 2}=2.0, \kappa=0.42$.

${ }^{2}$ In the continuity equation $\Gamma_{\text {eff }}=0$.

${ }^{3} P_{k}=\mu_{\mathrm{t}}\left[2\left(\frac{\partial \tilde{u}}{\partial x}\right)^{2}+2\left(\frac{\partial \tilde{v}}{\partial y}\right)^{2}+\left(\frac{\partial \tilde{u}}{\partial y}+\frac{\partial \tilde{v}}{\partial x}\right)^{2}\right]-\frac{2}{3} \bar{\rho} \tilde{k}\left(\frac{\partial \tilde{u}}{\partial x}+\frac{\partial \tilde{v}}{\partial y}\right)$

$-\frac{2}{3} \mu_{\mathrm{t}}\left(\frac{\partial \tilde{u}}{\partial x}+\frac{\partial \tilde{v}}{\partial y}\right)^{2}, G_{k}=-\frac{\mu_{\mathrm{t}}}{\sigma_{\rho} \bar{\rho}^{2}}\left[\frac{\partial \bar{p}}{\partial x} \frac{\partial \bar{\rho}}{\partial x}+\frac{\partial \bar{p}}{\partial y} \frac{\partial \bar{\rho}}{\partial y}\right], \mu_{\mathrm{t}}=\frac{C_{\mu} \bar{\rho} \tilde{k}^{2}}{\tilde{\varepsilon}}$.
} 
5. Dissipation rate of turbulent kinetic energy:

$$
\varphi=\tilde{\varepsilon}, \quad \tilde{S}_{\varphi}=\left(C_{1} P_{k}+C_{2} G_{k}-C_{3} \bar{\rho} \tilde{\varepsilon}\right) \frac{\tilde{\varepsilon}}{\tilde{k}} .
$$

6. Total enthalpy:

$$
\varphi=\tilde{h}, \quad \tilde{S}_{\varphi}=0 .
$$

7. Total enthalpy variance:

$$
\begin{aligned}
\varphi= & h^{\prime \prime 2} \\
\tilde{S}_{\varphi}= & C_{g, 1} \frac{\mu_{\mathrm{t}}}{\sigma_{g}}\left[\left(\frac{\partial \tilde{h}}{\partial x}\right)^{2}+\left(\frac{\partial \tilde{h}}{\partial y}\right)^{2}\right] \\
& -C_{g, 2} \bar{\rho} h^{\prime \prime 2} \frac{\tilde{\varepsilon}}{\tilde{k}} .
\end{aligned}
$$

8. Species mass fractions: ${ }^{4}$

$$
\varphi=\tilde{Y}_{i}, \quad S_{\varphi}=\bar{\rho} \tilde{S}_{i} .
$$

9. Species variances and covariances:

$$
\begin{aligned}
\varphi= & \widetilde{Y_{i}^{\prime \prime} Y_{j}^{\prime \prime}} \\
\tilde{S}_{\varphi}= & C_{g, 1} \frac{\mu_{\mathrm{t}}}{\sigma_{g}}\left[\frac{\partial \tilde{Y}_{i}}{\partial x} \frac{\partial \tilde{Y}_{j}}{\partial x}+\frac{\partial \tilde{Y}_{i}}{\partial y} \frac{\partial \tilde{Y}_{j}}{\partial y}\right] \\
& -C_{g, 2} \bar{\rho}\left(\widetilde{Y_{i}^{\prime \prime} Y_{j}^{\prime \prime}}\right) \frac{\tilde{\varepsilon}}{\tilde{k}}+\bar{\rho}\left(\widetilde{Y_{i}^{\prime \prime} S_{j}}\right)+\bar{\rho}\left(\widetilde{Y_{j}^{\prime \prime} S_{i}}\right) .
\end{aligned}
$$

10. Enthalpy-species covariances:

$$
\begin{aligned}
\varphi= & \widetilde{Y_{\mathrm{i}}^{\prime \prime} h^{\prime \prime}} \\
\tilde{S}_{\varphi}= & C_{g, 1} \frac{\mu_{\mathrm{t}}}{\sigma_{g}}\left[\frac{\partial \tilde{Y}_{i}}{\partial x} \frac{\partial \tilde{h}}{\partial x}+\frac{\partial \tilde{Y}_{i}}{\partial y} \frac{\partial \tilde{h}}{\partial y}\right] \\
& -C_{g, 2} \bar{\rho}\left(\widetilde{Y_{i}^{\prime \prime} h^{\prime \prime}}\right) \frac{\tilde{\varepsilon}}{\tilde{k}} .
\end{aligned}
$$

Turbulence closure is achieved by a two-layer $k-\varepsilon$ model [20,21]. In this model the flow domain is split into two zones. In the inner zone which includes the viscous sublayer, the buffer layer, and part of the fully turbulent layer only the $\tilde{k}$ equation is solved, $\tilde{\varepsilon}$ is calculated from $\tilde{\varepsilon}=\tilde{k}^{3 / 2} / \ell_{\varepsilon}$ and the turbulent viscosity from $\mu_{t}=\bar{\rho} C_{\mu} \tilde{k}^{1 / 2} \ell_{\mu}$, where $\ell_{\mu}$ and $\ell_{\varepsilon}$ are length scales containing damping effects in the near-wall region in terms of a local turbulence

$$
{ }^{4} \tilde{S}_{i}=W_{i} \sum_{k}\left(v_{i, k}^{\prime \prime}-v_{i, k}^{\prime}\right)\left(\frac{\tilde{\omega}_{k}}{\rho}\right) .
$$

Reynolds number $R_{k}=\rho \tilde{k}^{1 / 2} y / \mu$ with $y$ the distance from the wall [20]: $\ell_{\mu}=C_{\ell} y\left[1-\exp \left(-R_{k} / A_{\mu}\right)\right]$, $\ell_{\varepsilon}=C_{\ell} y\left[1-\exp \left(-R_{k} / A_{\varepsilon}\right)\right]$ with $C_{\ell}=\kappa C_{\mu}{ }^{-3 / 4}$, $A_{\varepsilon}=2 C_{\ell}$, and $A_{\mu}=70$. In the outer zone the standard two-equation $k-\varepsilon$ model is used, adopted as in [22] for variable density flows (Eqs. (6) and (7)). Both models are then merged at a location corresponding to $R_{k}=250$. Standard values are used for the turbulence constants [23] (see Footnote 1). The two-layer $k-\varepsilon$ turbulence model was adopted for two reasons. The first was the need to resolve the extended (for the Reynolds numbers of this study) viscous-dominated near-wall region and the second was the need of a near-wall turbulence model which is not as computationally intensive as the full two-equation low Reynolds number models [24]. The last point cannot be understated in CST which has the additional complication of gaseous and surface chemical reactions.

The thermochemistry modelling includes transport equations for the Favre-averaged mean values, variances, and covariances of the total enthalpy and the species mass fractions (Eqs. (8)-(12)). The approach of solving transport equations for all second order moments was originally developed by Bockhorn [25,26]. In Eqs. (8)-(12) gradient-type modelling has been used for the scalar fluxes and the dissipation hypothesis in a manner analogous to the turbulent flow model. To evaluate the mean chemical reaction rates (the terms $\tilde{S}_{i}$ in Eq. (10)) a presumed Gaussian joint p.d.f. was used as a coupling submodel for each elementary gaseous reaction. Measurements in non-catalytic turbulent reacting hydrogen-air boundary layers [27] and in non-reacting turbulent heated channel flows [28] have shown nearly symmetric scalar p.d.f.s, consistent with the Gaussian approach. In this respect, the Gaussian shape is less restrictive compared to open turbulent combustion applications [26]. For a bimolecular reaction the relevant joint p.d.f. is trivariate: $\tilde{P}\left(Y_{1}, Y_{2}, T\right)$ is the joint Gaussian p.d.f. depending on nine first and second order moments established by the temperature and the two species mass fractions. Tables of mean reaction rates were constructed for every elementary gas-phase reaction in terms of the nine controlling moments and a neural network scheme [29] was used to fit the data resulting in a speedy retrieval of these rates during the numerical solution procedure. Neural network fitting was also applied for the chemical reaction 
source terms in the transport equations for the species variances and covariances (terms $\widetilde{Y_{\mathrm{i}}^{\prime \prime}} S_{j}$ in Eq. (11)).

\subsection{Surface chemistry modelling}

The instantaneous form of the surface species coverage equations is

$$
\frac{\partial \Theta_{m}}{\partial t}=\sigma_{m} \frac{\dot{s}_{m}}{G}-\frac{\Theta_{m}}{G} \dot{G}, \quad m=1,2, \ldots, M_{\mathrm{s}} .
$$

For a surface reaction scheme that conserves the total number of surface sites $G$, as the one used in this study, the second term in the right-hand side of Eq. (13) is identically zero. The instantaneous interfacial boundary conditions for the gas-phase species are

$$
\begin{aligned}
G W_{k} \frac{\partial \Theta_{k}}{\partial t} & =-\left[\rho Y_{k}\left(\boldsymbol{v}+\boldsymbol{V}_{k}\right)\right]_{+} \cdot \boldsymbol{n}_{+}+\dot{s}_{k} W_{k}, \\
k & =1,2, \ldots, K_{\mathrm{g}} .
\end{aligned}
$$

with $\boldsymbol{n}_{+}$the unit vector normal to the catalyst surface and $\left(\boldsymbol{v}_{k}\right)_{+}=-\left(\left(\Gamma_{k} / Y_{k}\right)\left(\partial Y_{k} / \partial y\right)_{+}\right) \boldsymbol{n}_{+}$.

The left-hand side in Eq. (14) is the mass accumulation at the surface, the first term in the right-hand side is the combined Stefan and diffusive flux to the surface, and the second term is the surface reaction. For a statistically steady operation, averaging of Eqs. (13) and (14) removes the transient terms as well as the Stefan flux to the surface. The averaging, however, introduces the same difficulties with the chemical source terms $\dot{s}_{k}$ and $\dot{s}_{m}$ as with the gaseous reaction rates discussed previously. It can be argued that the catalyst, depending on its activity, can respond fast to fluctuations of the thermochemical field. However, the major non-linearity in the chemical source terms due to the Arrhenius exponential is removed since the wall temperature is fixed in this study. Moreover, in a technical application the presence of a catalyst support material with large thermal inertia diminishes the surface temperature fluctuations. In addition, the adsorption reactions are first-order with respect to the adsorbing species and hence they are a linear function of the relevant concentration; in this case averaging of the reaction rates produces (for fixed $T_{\mathrm{W}}$ ) exactly the mean concentration of the relevant species. In any case, even for reaction rates with non-linear concentration dependence the resulting effect of the non-linearity on the mean reaction rate is, for a fixed reaction temperature, small (see discussion in [25]). The mean heterogeneous reaction rate is then treated as a "laminar" one, evaluated at the corresponding mean values of the wall temperature and concentrations: in adsorption reactions, e.g., $\tilde{\tilde{s}}_{k}=\dot{s}_{k}\left(T_{\mathrm{W}}, Y_{k}, \tilde{\Theta}_{\mathrm{Pt}}\right)$, and for reactions involving only surface species, $\tilde{\dot{s}}_{m}=$ $\dot{s}_{m}\left(T_{\mathrm{W}}, \tilde{\Theta}_{i}\right)$ with $i$ the participating surface species.

\subsection{Boundary conditions}

At the catalytic wall $(y=H / 2)$ the no-slip condition is used for $\tilde{u}$ and $\tilde{v}, T=T_{\mathrm{W}}, \tilde{k}=0, \partial \tilde{\varepsilon} / \partial y=0$, and all the thermochemical fluctuations are set to zero. At the plane of symmetry $(y=0) \tilde{v}=0$ and $\partial \tilde{\varphi} / \partial y=0$ for the rest of the variables. Finally, at the exit plane $(x=L)$ the boundary conditions are $\tilde{v}=0$ and zero Neumann outflow conditions $(\partial \tilde{\varphi} / \partial x=0)$ for the other variables. The inlet conditions are uniform properties as stated in Section 2.

\subsection{Chemical kinetics}

For gaseous chemistry the full $\mathrm{H} / \mathrm{O}$ mechanism from Warnatz is used [30]; it includes 19 reversible reactions and eight species (excluding the carrier nitrogen). For surface chemistry the Langmuir-Hinshelwood $\mathrm{H}_{2} / \mathrm{O}_{2}$ scheme over Pt is employed from Deutchmann et al. [16]; it has 10 irreversible and three reversible reactions involving six gaseous and four surface species (excluding Pt). The surface reaction scheme is presented in Table 1. The thermodynamic data needed to evaluate the equilibrium constants for the three reversible surface reactions were taken from Warnatz et al. [31].

\subsection{Solution algorithm}

A SIMPLER-based finite volume procedure was used by solving iteratively the discretized algebraic gas-phase transport equations with an ADI algorithm [32]. A staggered grid of $100 \times 28$ points (in $x$ and $y$, respectively) with variable spacing in both directions was sufficient to produce numerically accurate results. Gravity was not included as it was found to have minimal influence for the Reynolds numbers of this study. Computations were then carried out over half the channel domain. Surface and gas-phase chemistries are 
Table 1

Elementary $\mathrm{H}_{2} / \mathrm{O}_{2}$ surface reaction scheme from $[16]^{\mathrm{a}}$

\begin{tabular}{lllll}
\hline Reaction & $A(\mathrm{~mol} \mathrm{cms})$ & $E_{\mathrm{a}}(\mathrm{kJ} / \mathrm{mol})$ & $\gamma$ & No. \\
\hline $\mathrm{H}_{2}+2 \mathrm{Pt}(\mathrm{s}) \rightarrow 2 \mathrm{H}(\mathrm{s})$ & & & 0.046 & $(1)$ \\
$2 \mathrm{H}(\mathrm{s}) \rightarrow \mathrm{H}_{2}+2 \mathrm{Pt}(\mathrm{s})$ & $3.7 \mathrm{E}+13$ & $67.4-6 \Theta_{\mathrm{H}}$ & 1.0 & $(2)$ \\
$\mathrm{H}+\mathrm{Pt}(\mathrm{s}) \rightarrow \mathrm{H}(\mathrm{s})$ & & & 0.07 & $(3)$ \\
$\mathrm{O}_{2}+\mathrm{Pt}(\mathrm{s}) \rightarrow \mathrm{O}(\mathrm{s})$ & & $213.2-60 \Theta_{0}$ & & $(4)$ \\
$2 \mathrm{O}(\mathrm{s}) \rightarrow \mathrm{O}_{2}+2 \mathrm{Pt}(\mathrm{s})$ & $3.7 \mathrm{E}+13$ & & 1.0 & $(5)$ \\
$\mathrm{O}+\mathrm{Pt}(\mathrm{s}) \rightarrow \mathrm{O}(\mathrm{s})$ & & 40.3 & 0.75 & $(6)$ \\
$\mathrm{H}_{2}+\mathrm{pt}(\mathrm{s}) \rightarrow \mathrm{H}_{2} \mathrm{O}(\mathrm{s})$ & & & 1.0 & $(7)$ \\
$\mathrm{H}_{2} \mathrm{O}(\mathrm{s}) \rightarrow \mathrm{H}_{2} \mathrm{O}+\mathrm{Pt}(\mathrm{s})$ & $1.0 \mathrm{E}+13$ & 192.8 & $(9)$ \\
$\mathrm{OH}+\mathrm{Pt}(\mathrm{s}) \rightarrow \mathrm{OH}(\mathrm{s})$ & & 11.5 & $(10)$ \\
$\mathrm{OH}(\mathrm{s}) \rightarrow \mathrm{OH}+\mathrm{Pt}(\mathrm{s})$ & $1.0 \mathrm{E}+13$ & 17.4 & $(11),(12)$ \\
$\mathrm{H}(\mathrm{s})+\mathrm{O}(\mathrm{s})=\mathrm{OH}(\mathrm{s})+\mathrm{Pt}(\mathrm{s})$ & $3.7 \mathrm{E}+21$ & 48.2 & $(13),(14)$ \\
$\mathrm{H}(\mathrm{s})+\mathrm{OH}(\mathrm{s})=\mathrm{H}_{2} \mathrm{O}(\mathrm{s})+\mathrm{Pt}(\mathrm{s})$ & $3.7 \mathrm{E}+21$ & $3.7 \mathrm{E}+21$ & & $(15),(16)$ \\
$\mathrm{OH}(\mathrm{s})+\mathrm{OH}(\mathrm{s})=\mathrm{H}_{2} \mathrm{O}(\mathrm{s})+\mathrm{O}(\mathrm{s})$ & & & \\
\hline
\end{tabular}

a (s) Denotes surface-bound species and $\gamma$ 's are sticking coefficients. The sticking coefficient of $\mathrm{O}_{2}$ (reaction (4)) is temperature dependent: $\gamma_{\mathrm{O}_{2}}(T)=0.07\left(T_{0} / T\right)$, with $T_{0}=300 \mathrm{~K}$. The order of $\mathrm{H}_{2}$ adsorption (reaction (1)) is unity with respect to platinum.

coupled via the interfacial boundary conditions. After an iteration for all gas-phase variables is completed, the averaged Eqs. (13) and (14) are solved simultaneously (in our previous laminar work $[14,15]$ these sets were solved sequentially) using a modified Newton's method. The Chemkin database [33] was used to evaluate gaseous thermodynamic and transport properties and the Surface Chemkin [34] to evaluate surface reaction rates. The computational time was about $12 \mathrm{~h}$ on a DEC4130 machine. With only the surface chemistry present, the corresponding time was reduced to $6 \mathrm{~h}$.

\section{Results and discussion}

The conditions of this study are presented in Table 2. There are three basic cases denoted as (a), (b) and (c) with equivalence ratios $0.240,0.225$, and 0.215 ,

Table 2

Summary of conditions ${ }^{\mathrm{a}}$

\begin{tabular}{lll}
\hline Case & Equivalence ratio $(\varphi)$ & Subcases \\
\hline (a) & 0.240 & CG, C, L \\
(b) & 0.225 & CG, C, L \\
(c) & 0.215 & CG, C, L \\
\hline
\end{tabular}

${ }^{a}$ CG: turbulent with catalytic and gaseous reactions. C: turbulent, only catalytic reactions. L: laminar, with catalytic and gaseous reactions. respectively. The difference in equivalence ratios is sufficient for the ensuing discussion. For each case three different subcases were examined: subcases CG are turbulent with both catalytic and gaseous reactions, subcases $\mathrm{C}$ are turbulent with only catalytic reactions (gaseous chemistry is switched-off), and finally the subcases $\mathrm{L}$ are laminar with both catalytic and gaseous reactions. In the $\mathrm{L}$ cases the same uniform inlet conditions and computational grid were used as in the corresponding turbulent CG cases, but turbulence was turned off: transport equations are solved only for the mean properties (the governing equations for the laminar case were presented in our earlier work [14]).

\subsection{Turbulent heterogeneous-homogeneous combustion}

Transverse profiles of Favre-averaged temperatures and hydrogen mass fractions (the wall is located at $y=5 \mathrm{~mm}$ ) are illustrated in Fig. 2 at four selected streamwise distances. Fig. 2 is discussed with the aid of Fig. 3 presenting streamwise profiles of catalytic and gaseous hydrogen conversion rates. The catalytic conversion refers to only one plate. The volumetric gaseous conversion rate of Fig. 3 has been integrated over the channel half-width so that it can be directly compared to the catalytic surface rate. The catalytic fuel conversion rate is maximum at the channel entrance since the mass transport coefficients are the 


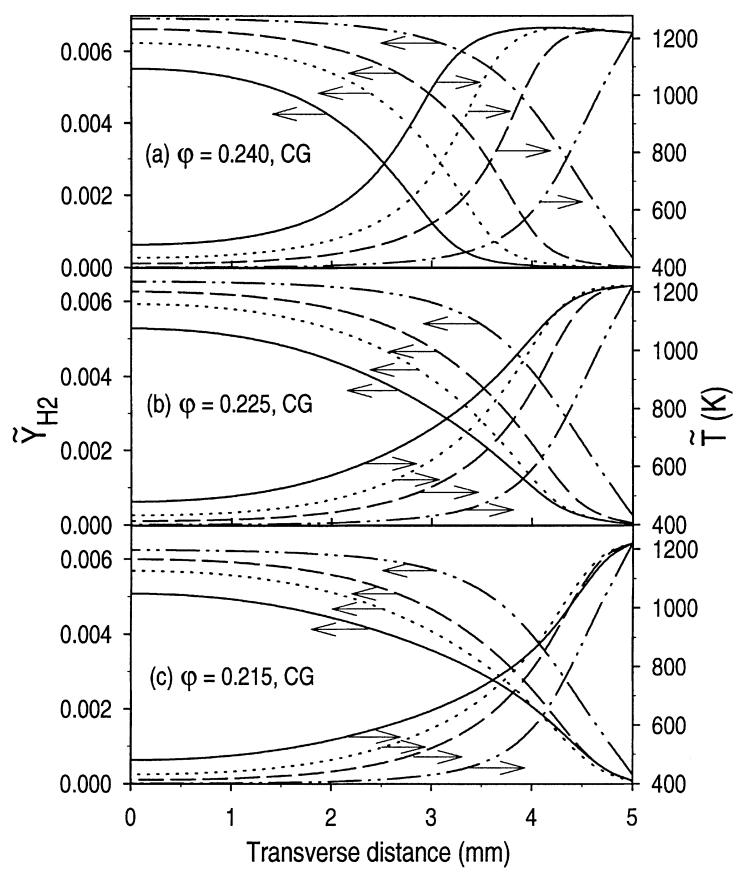

Fig. 2. Transverse profiles of Favre-averaged temperatures and hydrogen mass fractions at four selected streamwise distances, for three different turbulent cases with both catalytic and surface reactions (CG cases). Streamwise distances: $x=25 \mathrm{~mm}$ (dash-double dotted), $x=61 \mathrm{~mm}$ (dashed), $x=98 \mathrm{~mm}$ (dotted), and $x=150 \mathrm{~mm}$ (solid) lines. The wall is located at $y=5 \mathrm{~mm}$.

highest there and at the same time the surface reactions are close to their mass-transport limit (heterogeneous ignition achieved at the channel entry) given the high wall temperature of this study. The catalytic conversion drops downstream due to reduced mass transport coefficients and upstream fuel depletion. Homogeneous ignition occurs at $x \approx 25 \mathrm{~mm}$ in all cases as illustrated in Fig. 3. The relative insensitivity of the ignition distance on moderate variations of the equivalence ratio is consistent with experimental observations [15] and with theoretical asymptotic approaches [35] where the homogeneous ignition distance is shown to scale as $1 / Y_{\mathrm{F}, \mathrm{IN}}^{n_{\mathrm{F}}}$ with $Y_{\mathrm{F}, \mathrm{IN}}$ the incoming fuel mass fraction and $n_{\mathrm{F}}$ a global fuel reaction-rate order, which is typically a small exponent. Following homogeneous ignition the gaseous conversion increases rapidly in case (a) (see Fig. 3a), although both modes of conversion are equally important in the range $30 \mathrm{~mm}<x<40 \mathrm{~mm}$, where significant fuel leakage occurs through the gaseous reaction zone and

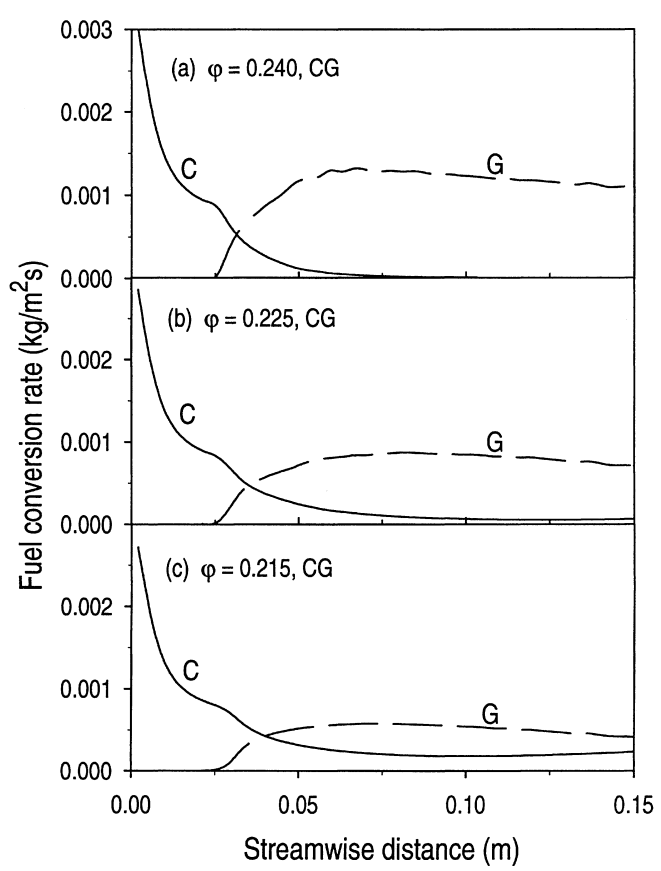

Fig. 3. Streamwise profiles of catalytic (C) and gaseous (G) fuel conversion rates, for three turbulent $\mathrm{CG}$ cases. The volumetric gaseous fuel conversion rate has been integrated over the channel half-width.

the escaping fuel is consumed heterogeneously at the surface. Gaseous combustion becomes the dominant fuel conversion mode for $x>60 \mathrm{~mm}$. The last point is also illustrated in Fig. 2a, where the transverse hydrogen wall gradient is zero for the streamwise distances $x=98$ and $150 \mathrm{~mm}$. In addition, the gaseous combustion is confined close to the catalytic surface. At the channel exit $(x=150 \mathrm{~mm})$ Fig. 2a indicates that the combustion wave has propagated only about $2 \mathrm{~mm}$ from the wall. This is due to the diffusional imbalance of the hydrogen $(L e<1)$ : fuel is transported towards the surface more effectively than heat away from it resulting in confinement of the combustion wave close to the surface. This effect has been shown in the catalytic stagnation-point flow studies of Law and Sivashinsky [36] and confirmed in the laminar hydrogen channel CST experiments of Buser et al. [11]. The temperature profiles in Fig. 2a have a small but negative transverse wall gradient at the locations downstream homogeneous ignition $(x>25 \mathrm{~mm})$ indicating heat transfer from the gas towards the wall. The leaner cases (b) and (c) exhibit qualitative differences 
compared to case (a). Figs. $2 \mathrm{~b}$ and $3 \mathrm{~b}$ indicate that the gaseous combustion in case (b) is confined closer to the wall and that a small catalytic conversion persists well after homogeneous ignition down to the channel exit. In case (c) gaseous combustion is concentrated even closer to the wall and both the catalytic and the gaseous modes are important for fuel conversion. The catalytic fuel conversion in case (c) is actually increasing for $x>100 \mathrm{~mm}$. Finally, in the leaner cases (b) and (c), the temperature wall gradients remain positive after ignition as illustrated in Fig. $2 \mathrm{~b}$ and $\mathrm{c}$.

To understand the interaction of combustion and turbulence responsible for the above processes, the transverse extent of the gaseous reaction is firstly delineated. Fig. 4 presents transverse profiles of the local Favre-average hydrogen reaction rate for the three streamwise locations of Fig. 2 that are downstream the homogeneous ignition point. The successive approach of the gaseous combustion zone towards the wall with mixture leaning is evident in Fig. 4. A small amount of incomplete gaseous combustion occurs in

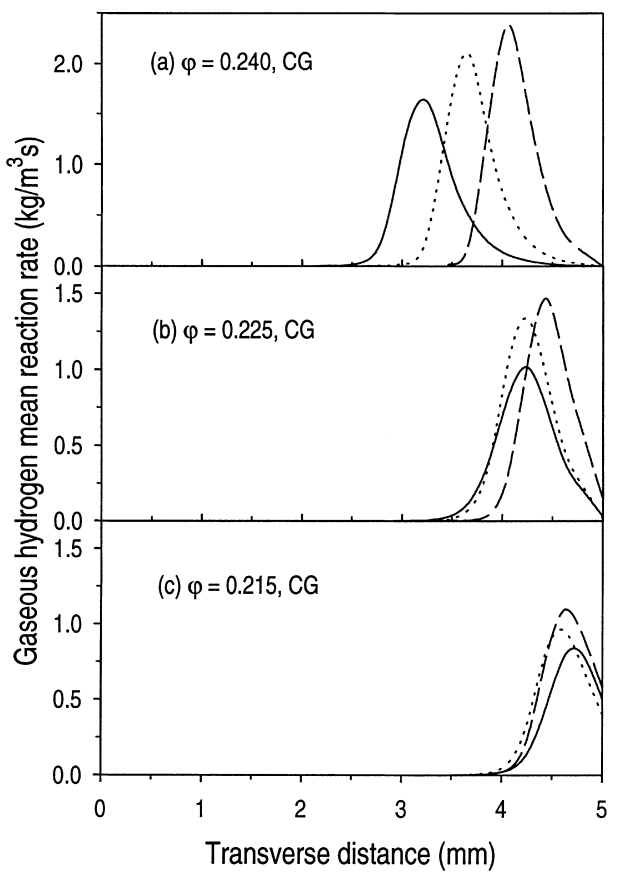

Fig. 4. Transverse profiles of Favre-averaged hydrogen gaseous reaction rates for three selected streamwise distances downstream homogeneous ignition, CG cases. The notation of the streamwise distances is the same as in Fig. 2. case (b) while in case (c) the reaction zone has been pushed against the catalytic surface. There is then significant fuel leakage through this zone resulting in the appreciable catalytic conversion discussed in Fig. 3c. Turbulence affects gaseous combustion through the increased turbulent transport and the induced thermochemical fluctuations. The effect of the thermochemical fluctuations is discussed first. Fig. 5 presents normalized Favre-averaged root mean square (rms) temperature fluctuations. The vertical bars in Fig. 5 define the transverse extent of the gaseous reaction zone (calculated with the aid of Fig. 4) for the three $x$-locations downstream homogeneous ignition; 95\% of gaseous combustion has been completed in the zones defined by the vertical bars and the wall. The rms temperature profiles in Fig. 5a peak near the point of maximum mean temperature gradient and at a distance from the wall which increases with increasing $x$.

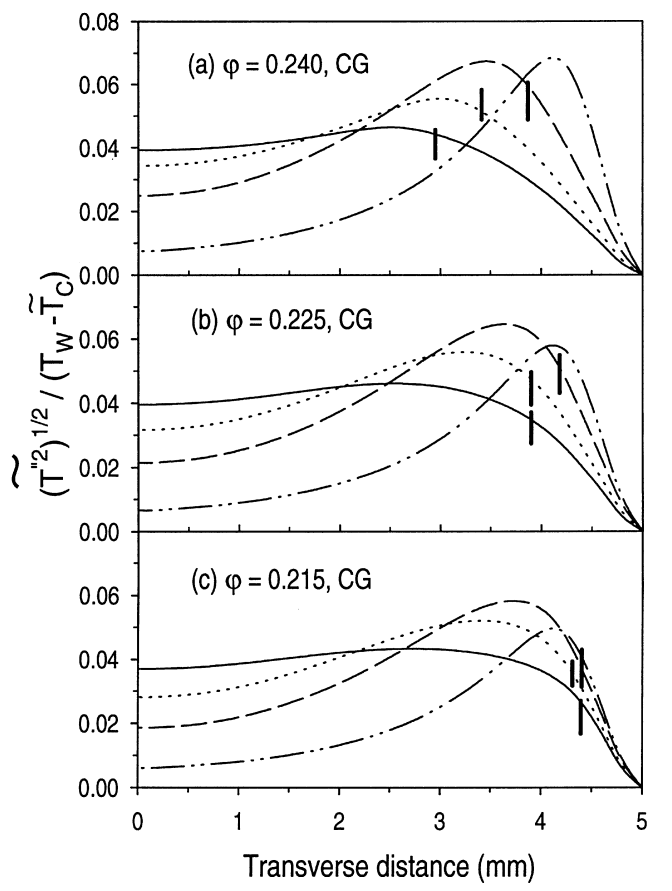

Fig. 5. Transverse profiles of normalized Favre-averaged rms temperature fluctuations at four selected streamwise distances, CG cases. $\tilde{T}_{\mathrm{C}}$ denotes the temperature at the plane of symmetry $(y=0)$. The vertical bars indicate the transverse extent of the gaseous reaction zone from the wall for the three $x$-locations downstream homogeneous ignition. The notation of the streamwise distances is the same as in Fig. 2. 
The peak levels of the intensities $\left(\widetilde{T^{\prime \prime 2}}\right)^{1 / 2} /\left(T_{\mathrm{W}}-\tilde{T}_{\mathrm{C}}\right)$ are as high as $7 \%$ at $x=61 \mathrm{~mm}$ and drop downstream. Such intensity levels are typical in turbulent boundary layer combustion [27] or turbulent heat transfer in channels [28]. In addition, the peak rms temperature fluctuations are always located outside the reaction zone. For example, in Fig. 5a the peak rms temperature fluctuations at $x=61 \mathrm{~mm}$ are about $1.5 \mathrm{~mm}$ away from the wall while the reaction zone extends up to $1.2 \mathrm{~mm}$ from it. Moreover, the peak reaction rate is located about $0.9 \mathrm{~mm}$ from the wall. Temperature fluctuations still persist in the reaction zone but with diminishing magnitude as the wall is approached. Fig. 5a-c indicate that mixture leaning results in small reduction of the rms fluctuation levels and successive approach of the locations of peak rms fluctuations closer to the wall. In all cases, however, the peak rms fluctuations lie outside the extent of the gaseous reaction zone; this is also the case for all other scalar rms fluctuations. The previous findings have qualitative similarities to experimental observations [27], although the latter refer to non-catalytic turbulent hydrogen-air boundary layer combustion over a constant temperature flat plate. They report that mixture leaning results in a small decrease of measured rms density fluctuations and a successive approach of the locations of peak rms density fluctuations closer to the wall. The levels and location of the thermochemical fluctuations have a direct consequence on the gaseous combustion processes. Temperature fluctuations increase the mean gaseous reaction rate, and this is generally the case for all thermochemical fluctuations [25]. Given the fact that the fluctuation levels are low inside the reaction zone, they have only a moderate influence on the mean gaseous reaction rates. This outcome has to be examined in conjunction with the turbulent transport to the reaction zone discussed next.

Normalized turbulent intensities $\tilde{k}^{1 / 2} / \tilde{U}_{\mathrm{C}}$ are presented in Fig. 6. Turbulence intensities are substantially suppressed by combustion (increasing $\varphi$ ). At $x=150 \mathrm{~mm}$, e.g., the peak turbulent intensities are in cases (b) and (c) higher by 35 and 50\%, respectively, compared to case (a). In addition the points of peak $\tilde{k}$ move only slightly closer to the wall with mixture leaning. The suppression of the turbulent kinetic energy is due to the relaminarization induced by the gaseous heat release. The sensitivity of turbulence

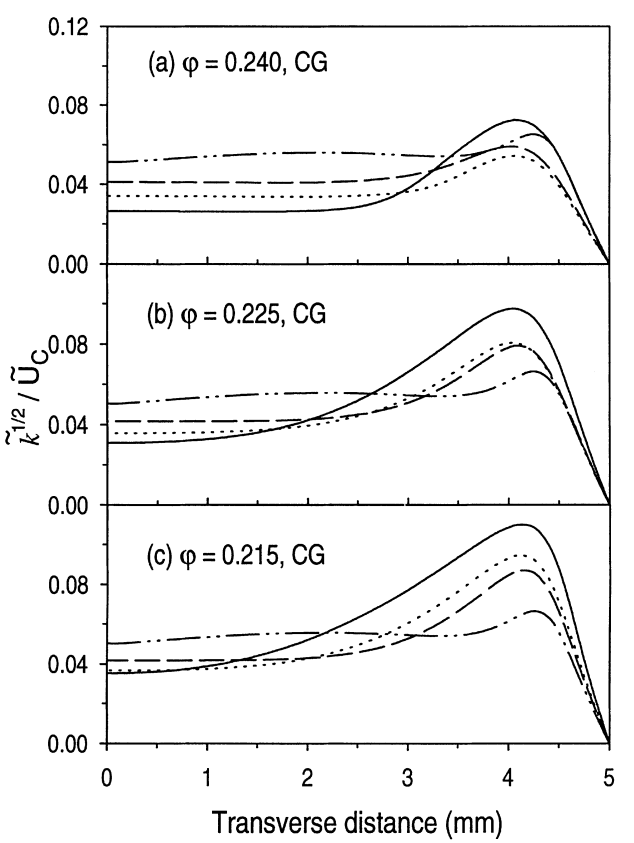

Fig. 6. Transverse profiles of normalized turbulent kinetic energy at four selected streamwise distances, CG cases. $\tilde{U}_{\mathrm{C}}$ denotes the axial velocity at the plane of symmetry $(y=0)$. The notation of the streamwise distances is the same as in Fig. 2.

intensity with mixture leaning is in good agreement with turbulent boundary layer combustion observations [27]; they measure a nearly fivefold suppression of the streamwise velocity fluctuations with an increase of equivalence ratio from 0.1 to 0.2 . The suppression of the turbulent kinetic energy and the insensitivity of its peak location to mixture strength imply that at a given distance from the wall the leaner cases have higher turbulent transport coefficients $\left(\mu_{\mathrm{t}}=\bar{\rho} C_{\mu} \tilde{k}^{1 / 2} \ell_{\mu}\right)$. The higher transport coefficients coupled with the slower reaction rates of the leaner cases and the mild influence of the thermochemical fluctuations on the mean reaction rates result in a push of the reaction zone closer to the wall and finally to fuel leakage and incomplete combustion. Incomplete combustion can be more pronounced at the downstream locations as the mixture strength has been reduced due to upstream depletion (see Fig. 3c). The foregoing have a direct analogy to catalytic laminar stagnation point flow studies [36]: increasing the strain rate leads, for $L e<1$, to confinement of the reaction zone closer to the wall, incomplete 


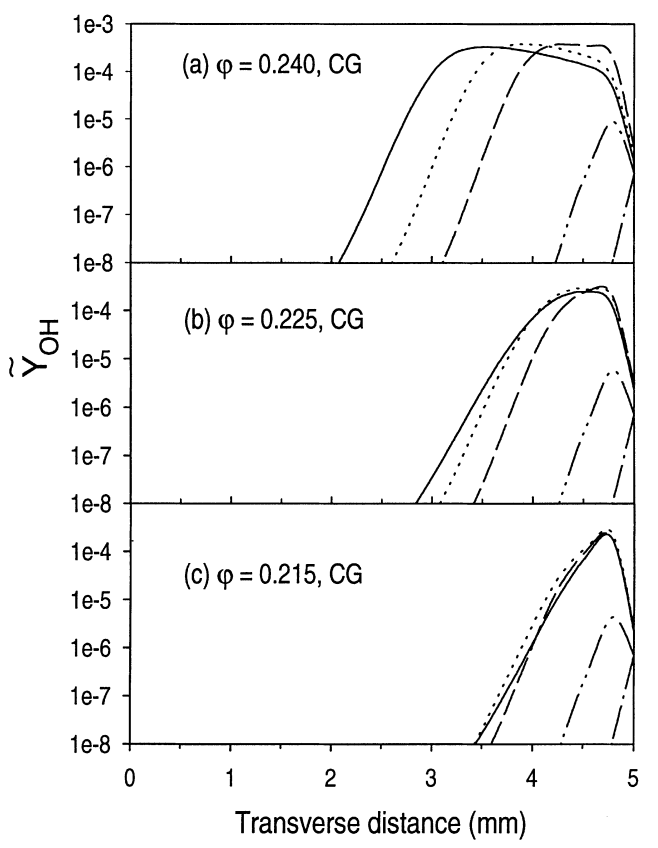

Fig. 7. Transverse profiles of Favre-averaged $\mathrm{OH}$ mass fractions at five selected streamwise distances, CG cases. The notation of the streamwise locations is the same as in Fig. 2, with the addition of $x=10 \mathrm{~mm}$ (dash-dotted lines).

gaseous combustion followed by subsequent catalytic conversion of the leaked reactant, and finally to extinction.

Radicals are important for homogeneous ignition and their near-wall levels are determined by the coupling between gaseous and heterogeneous reactions. Favre-averaged profiles of the $\mathrm{OH}$ mass fraction are presented in Fig. 7; an additional streamwise location before homogeneous ignition $(x=10 \mathrm{~mm})$ is included in this figure. The $\mathrm{OH}$ wall gradients are positive (net-desorptive) at $x=10 \mathrm{~mm}$ and negative (net-adsorptive) at the other streamwise distances. The transition from desorptive to adsorptive $\mathrm{OH}$ wall fluxes takes place at $x \approx 16 \mathrm{~mm}$, that is well before homogeneous ignition. Hence, gas-phase reactions precede any significant gaseous heat release or fuel conversion. This effect has been also observed in methane catalytic combustion computations [14] and signifies the importance of a detailed surface reaction scheme in accurately predicting the coupling between gaseous and surface reactions in the pre-ignition period. Transverse profiles of the $\mathrm{H}$ radical are plotted in Fig. 8. The $\mathrm{H}$ profiles are always net-adsorptive due

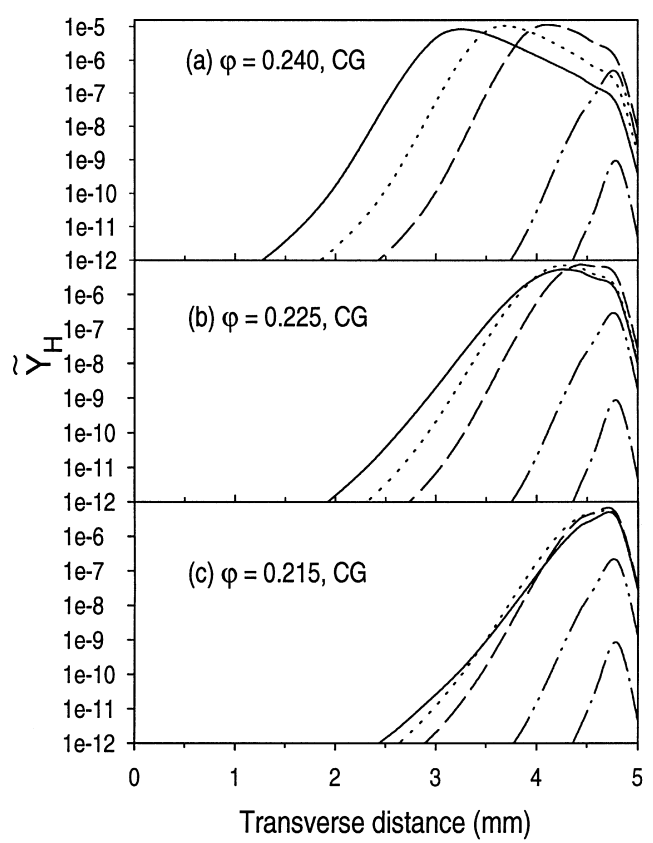

Fig. 8. Transverse profiles of Favre-averaged $\mathrm{H}$ mass fractions at five selected streamwise distances, CG cases. The notation is the same as in Fig. 7.

to the absence of any $\mathrm{H}$-desorption reactions in the heterogeneous reaction scheme (see Table 1). Hence, the catalytic wall is always a sink for the gaseous $\mathrm{H}$ radicals (the same is also the case for the $\mathrm{O}$ radicals).

To complete the picture of catalytic combustion inside the channel the surface coverage is presented for case (a) in Fig. 9. The coverage in cases (b) and (c) are similar and hence not repeated. The surface is covered primarily with platinum and oxygen over its entire length. For the fixed and high wall temperature of this study, heterogeneous ignition is achieved already from the channel entry as manifested by the large free platinum surface coverage at $x \approx 0$. At the channel entry the coverage of $\mathrm{O}(\mathrm{s})$ and $\mathrm{Pt}(\mathrm{s})$ are 0.433 and 0.565 , respectively, while at the channel exit the corresponding numbers are 0.517 and 0.481 , respectively. Reactions involving only surface species are very fast resulting in very low values for the $\mathrm{H}(\mathrm{s})$. Moreover, the $\mathrm{H}(\mathrm{s})$ coverage drops sharply after homogeneous ignition $(x \approx 25 \mathrm{~mm})$ due to the corresponding decrease in the gaseous $\mathrm{H}_{2}$ concentration near the wall. The coverage of $\mathrm{H}_{2} \mathrm{O}(\mathrm{s})$ and $\mathrm{OH}(\mathrm{s})$ drop with increasing $x$ resulting in the aforementioned increase of $\mathrm{O}(\mathrm{s})$. 


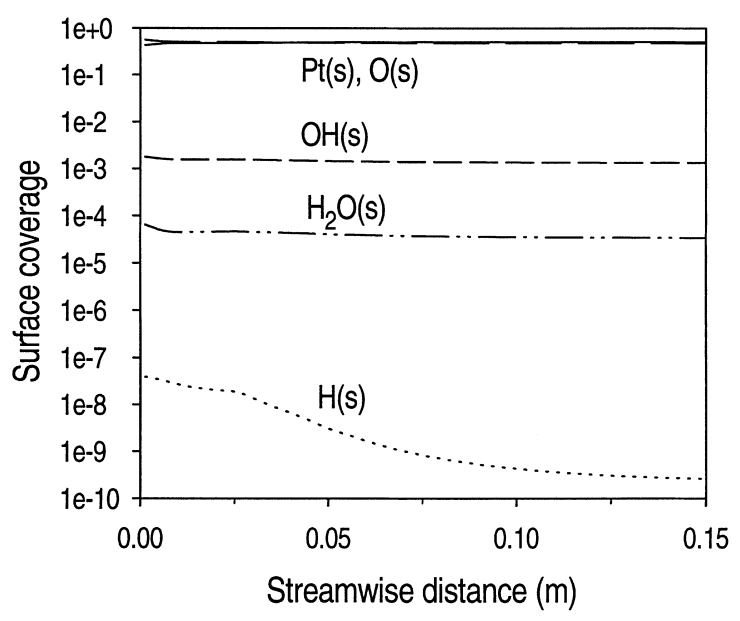

Fig. 9. Streamwise profiles of surface coverage for case (a), CG.

\subsection{Turbulent heterogeneous combustion}

The main influence of turbulence on purely catalytic combustion ( $\mathrm{C}$ cases) is the enhancement of the transport coefficients towards-or-away from the wall. The removal of gaseous reactions simplifies considerably the problem. Results are presented then for case (a) only, as all cases bear direct similarities. Transverse profiles of Favre-average temperatures and hydrogen mass fractions are presented in Fig. 10 and the stream-

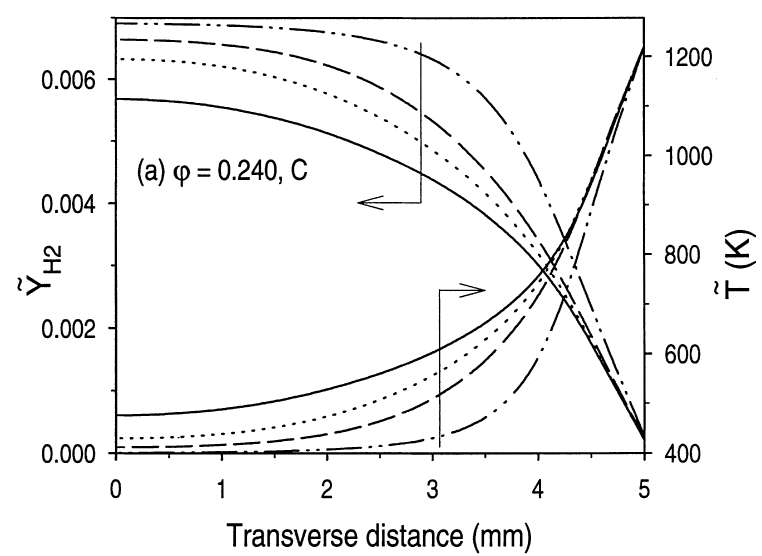

Fig. 10. Transverse profiles of Favre-averaged temperatures and hydrogen mass fractions at four selected streamwise distances, for case (a), with only catalytic reactions ( $\mathrm{C}$ case). The notation of the streamwise distances is the same as in Fig. 2.

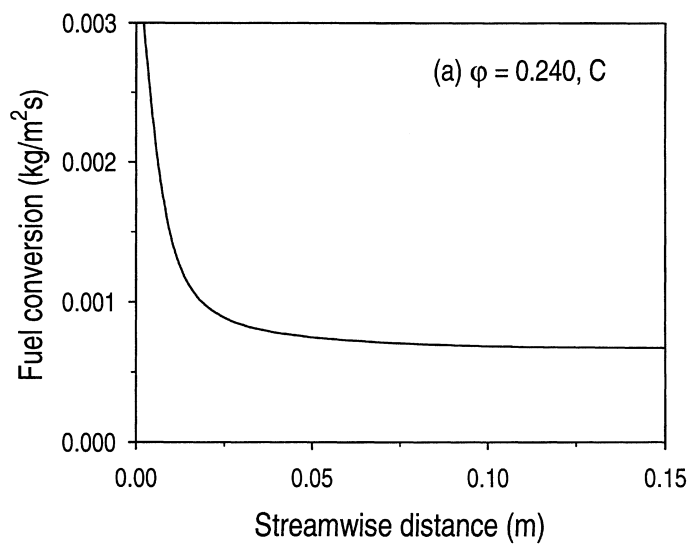

Fig. 11. Streamwise profile of the catalytic fuel conversion rate for case (a), C.

wise profile of catalytic conversion in Fig. 11. The catalytic conversion of Fig. 11 coincides with the corresponding catalytic conversion of the CG case (see Fig. 3a) down to the point of homogeneous ignition. Some finite rate effects are evident in Fig. 10. At the catalyst surface the hydrogen mass fraction is always non-zero, e.g., at $x=150 \mathrm{~mm}$ the surface concentration is about $5 \%$ of the symmetry plane concentration. Finally the absence of gaseous reactions results in large and positive transverse temperature wall gradients (Fig. 10).

In Fig. 12 the normalized rms temperature fluctuations, normalized turbulent intensities, and average $\mathrm{OH}$ mass fractions are presented for case (a). The locations of the peak rms temperature fluctuations are closer to the wall and their magnitudes are larger compared to the CG case of Fig. 5a. This is in accord with the turbulent boundary measurements of [27] in their experiments with complete gaseous combustion suppression ( $\varphi=0$, pure heat transfer mode). In addition, the turbulent kinetic energy increases substantially in comparison to the $\mathrm{CG}$ case (Fig. 6a). Finally the $\mathrm{OH}$ profiles of Fig. 12 are always net-desorptive with much lower near-wall $\mathrm{OH}$ levels compared to the CG case (Fig. 7a) due to the absence of gas-phase reactions. On the other hand, near the plane of symmetry the $\mathrm{OH}$ levels in the $\mathrm{C}$ case are higher than the corresponding ones of the CG case due to the absence of gaseous radical recombination reactions which are important in these colder zones. 


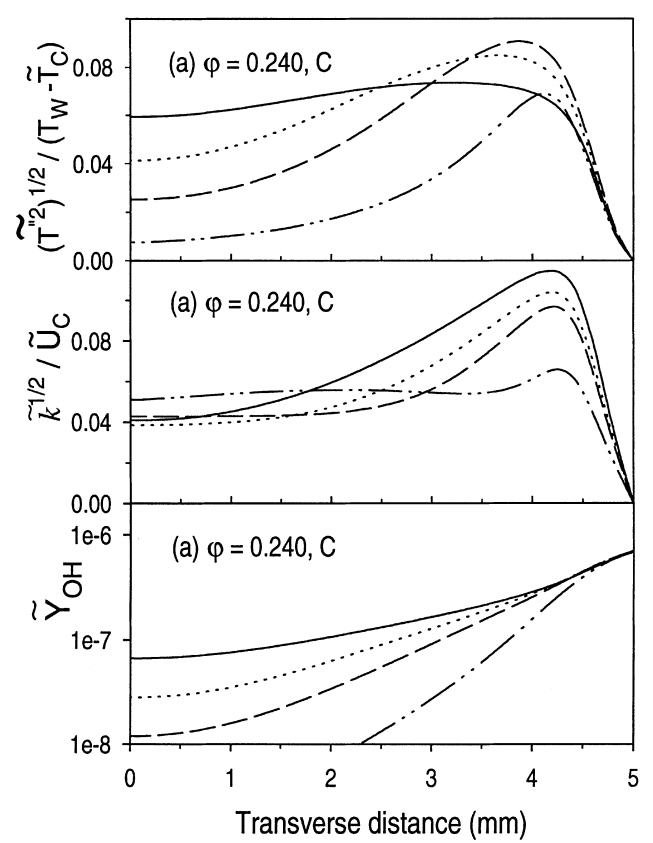

Fig. 12. Transverse profiles of normalized Favre-averaged rms temperature fluctuations, normalized turbulent kinetic energies, and Favre-average $\mathrm{OH}$ mass fractions for case (a), C. The notation of the streamwise distances is the same as in Fig. 2.

\subsection{Laminar heterogeneous-homogeneous combustion}

Of particular importance in CST is the assessment of performance differences between the laminar and turbulent flow modes. Comparisons between turbulent and laminar flow conditions are presented next. Transverse profiles of temperature and hydrogen mass fraction are presented in Fig. 13 for the three laminar cases. The catalytic and gaseous fuel conversions for the laminar (dotted lines) as well as the corresponding turbulent (solid lines) conditions are presented in Fig. 14. In all cases the turbulent catalytic fuel conversion rate is higher than the corresponding laminar rate and their difference increases with increasing streamwise distance. This is because turbulent mass transport coefficients are higher and surface reactions are still close to their mass-transport limit. Of more importance is, however, that homogeneous ignition comes about $5 \mathrm{~mm}$ earlier in the laminar cases. It is then clear that turbulence inhibits homogeneous ignition. This happens despite the fact that the turbulent cases

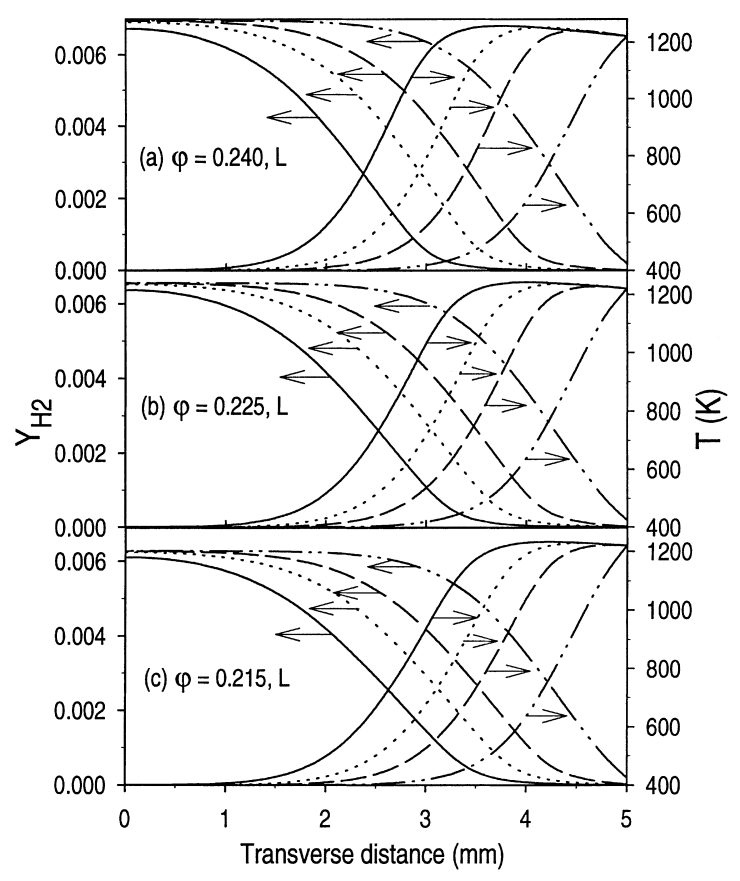

Fig. 13. Transverse profiles of temperatures and hydrogen mass fraction for the laminar cases. The notation of the streamwise distances is the same as in Fig. 2.

have higher near-wall hydrogen levels at all streamwise distances before homogeneous ignition. There are two reasons responsible for the higher near-wall hydrogen in the turbulent cases: the first is that the laminar boundary layer is thicker than the corresponding turbulent boundary layer and the second is the somewhat higher hydrogen wall concentrations in the turbulent case due to more pronounced finite rate surface effects induced by the increased surface loading. For example, at $x=20 \mathrm{~mm}$ the hydrogen wall concentrations in the turbulent CG cases (a), (b) and (c) are $2.827 \times 10^{-4}, 2.722 \times 10^{-4}$, and $2.639 \times 10^{-4}$, respectively, while the corresponding laminar concentrations are $2.544 \times 10^{-4}, 2.450 \times 10^{-4}$, and $2.374 \times 10^{-4}$. The homogeneous ignition inhibition is, as explained below, a result of increased heat transport away from the catalytic surface.

The transverse temperature profiles in Fig. 13 are steeper compared to Fig. 2, resembling more to a flame structure. In all laminar cases, within $1 \mathrm{~mm}$ from the symmetry plane $(y=0)$ the heat wave has not been sensed and temperatures are around $400 \mathrm{~K}$. 


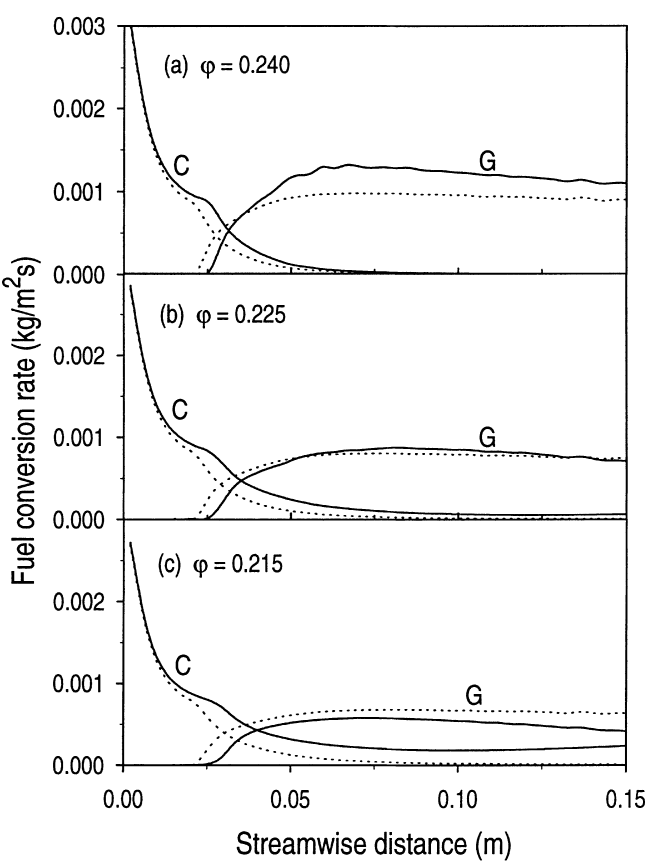

Fig. 14. Streamwise profiles of catalytic (C) and gaseous (G) fuel conversion rates for the laminar (dotted lines) and the corresponding turbulent (solid lines) cases. The volumetric gaseous fuel conversion rates have been integrated over the channel half-width.

Hydrogen depletion is, however, readily observable at the plane of symmetry, a result of the more efficient molecular mass transport. The turbulent profiles of Fig. 2, on the other hand, indicate a maximum $100 \mathrm{~K}$ temperature rise at the symmetry plane since turbulent transport tends to balance the effective heat and mass transport coefficients. Heat is transported more effectively in the turbulent case from the near-wall zone to the colder channel core resulting in homogeneous ignition inhibition. The difference in flow residence times for the near-wall gas could also contribute to the above inhibition. However, this effect is not as important since for the short development distances down to homogeneous ignition the streamwise velocities in the turbulent cases are only slightly higher that the corresponding laminar ones. The turbulent CST performance of Fig. 14 could be desirable in practical applications where increased heterogeneous conversion is sought with late homogeneous ignition (or no homogeneous ignition at all if a post-catalyst combustion zone is added) in order to reduce pollutant formation. Although the onset of gaseous combustion is delayed in the turbulent cases, it overtakes shortly the corresponding laminar gaseous conversion rates in cases (a) and (b). In all laminar cases there is no catalytic conversion at distances well downstream the homogeneous ignition location. In the leaner case (c) the gaseous turbulent reaction rate is lower than the corresponding laminar one due to fuel leakage; the combined catalytic and gaseous conversion is, however, higher in the turbulent case.

\section{Conclusions}

A two-dimensional elliptic model has been developed for the numerical investigation of turbulent catalytically stabilized (CST) combustion in plane channel flows. The model is capable of treating elementary heterogeneous and homogeneous chemical reactions. Application is made to turbulent CST of lean hydrogen-air mixtures at atmospheric pressures. The channel walls were coated with platinum and were held at a fixed temperature of $1220 \mathrm{~K}$. The following are the key conclusions.

1. The homogeneous combustion zone is confined close to the catalytic wall due to the diffusional imbalance of hydrogen. Mixture leaning results in a small decrease of peak rms temperature levels and in a successive approach of their location closer to the catalytic wall. Complete suppression of gaseous combustion increases the peak rms temperature levels and moves their location even closer to the wall.

2. The peak rms temperature fluctuations (as well as the other thermochemical fluctuations) are always located outside the extent of the gaseous reaction zone resulting in a small influence of thermochemical fluctuations on gaseous combustion.

3. Turbulence is significantly suppressed by gaseous combustion resulting in higher transport coefficients for the leaner mixtures, a push of the gaseous reaction zone towards the wall, incomplete gaseous combustion, and subsequent catalytic conversion of the leaked fuel. Both catalytic and gaseous modes are then important for the fuel conversion.

4. The surface is covered primarily with platinum and oxygen over its entire length. In addition, the hydrogen surface coverage drops substantially downstream the homogeneous ignition location. 
5. Comparison with corresponding laminar cases shows that turbulence inhibits homogeneous ignition due to the increased heat transport from the near-wall zone to the channel core.

\section{Nomenclature}

$C_{\mu}, C_{1}, C_{2}$,
$C_{3}, C_{g, 1}, C_{g, 2}$
$G$
$h$
$H$
$k$
$K_{\mathrm{g}}$
$L$
$L_{e}$
$M_{\mathrm{S}}$
$p$
$R_{k}$
$S_{\varphi}$
$S_{i}$
$s_{m}$
$T$
$U, u$
$v$
$V$
$W_{k}$
$x$
$y$
$Y_{i}$

$\begin{array}{ll}\text { Greek symbols } & \\ \Gamma & \begin{array}{l}\text { laminar transport coefficient } \\ \text { effective turbulent transport } \\ \text { coefficient, Eq. (2) } \\ \Gamma_{\text {eff }}\end{array} \\ \varepsilon & \begin{array}{l}\text { dissipation rate of turbulent kinetic } \\ \text { energy }\left(\mathrm{m}^{2} / \mathrm{s}^{3}\right)\end{array} \\ \Theta_{m} & \text { surface coverage of species } m \\ \kappa & \text { turbulence von Karman constant } \\ \mu & \text { laminar viscosity }(\mathrm{kg} / \mathrm{m} \mathrm{s}) \\ \mu_{\mathrm{t}} & \text { turbulent viscosity }(\mathrm{kg} / \mathrm{m} \mathrm{s}) \\ v_{i, k}^{\prime}, v_{i, k}^{\prime \prime} & \begin{array}{l}\text { stoichiometric coefficients of species } \\ i \text { in reaction } k\end{array}\end{array}$

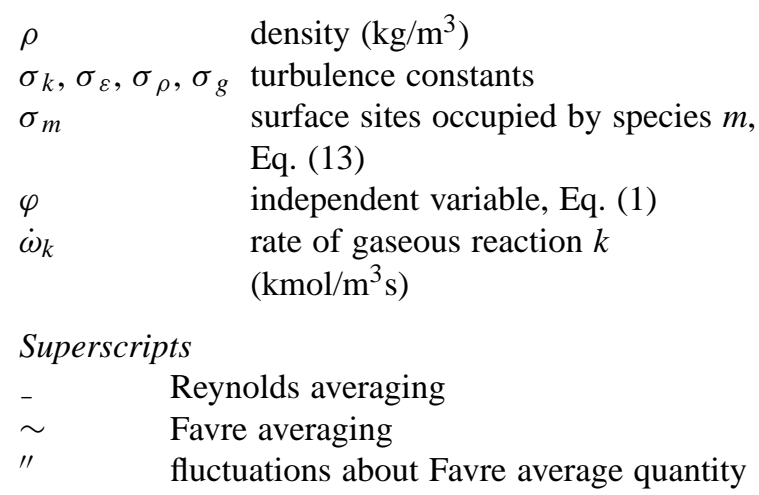

\section{Acknowledgements}

Support for this work was provided by the Swiss Federal Department of Energy (BFE) under contract No. 59048. We wish to thank Professor H.B. Bockhorn of Karlsruhe University for providing the SIMPLER-based non-reacting code over which the turbulent CST code of this study was built. We also acknowledge the help of Dr. C. Frouzakis from ETH Zuerich in the neural network fitting scheme.

\section{References}

[1] I. Stambler, Catalytic combustor design limits $\mathrm{NO}_{x}$ to less than 0.5 ppm, Gas Turbine World 23 (3) (1993)

[2] A. Schlegel, P. Benz, T. Griffin, W. Weisenstein, H. Bockhorn, Catalytic stabilization of lean premixed combustion: method for improving $\mathrm{NO}_{x}$ emissions, Combust. Flame 105 (1996) 332.

[3] T. Griffin, W. Weisenstein, A. Schlegel, S. Buser, P. Benz, H. Bockhorn, F. Mauss, Investigation of the $\mathrm{NO}_{x}$ advantage of catalytic combustion, in: Proceedings of the International Workshop on Catalytic Combustion, Tokyo, April 18-20, 1994, p. 138.

[4] X. Song, W.R. Williams, L.D. Schmidt, R. Aris, Ignition and extinction of homogeneous-heterogeneous combustion: $\mathrm{CH}_{4}$ and $\mathrm{C}_{3} \mathrm{H}_{8}$ oxidation on $\mathrm{Pt}$, in: Proceedings of the 23rd International Symposium on Combustion, The Combustion Institute, 1990, p. 1129. 
[5] H. Ikeda, P.A. Libby, F.A. Williams, J. Sato, Catalytic combustion of hydrogen-air mixtures in stagnation flows, Combust. Flame 93 (1993) 138.

[6] P.A. Bui, D.G. Vlachos, P.R. Westmoreland, Homogeneous ignition of hydrogen/air mixtures over platinum, in: Proceedings of the 26th International Symposium on Combustion, The Combustion Institute, 1996, p. 1763.

[7] R.W. Schefer, Catalyzed combustion of $\mathrm{H}_{2}$ /air mixtures in a flat plate boundary layer: II. Numerical model, Combust. Sci. Technol. 45 (1982) 171.

[8] T.A. Griffin, L.D. Pfefferle, M.J. Dyer, D.R. Crosley, The ignition of methane/ethane boundary layer flows by heated catalytic surfaces, Combust. Sci. Technol. 65 (1989) 19.

[9] P. Markatou, L.D. Pfefferle, M.D. Smooke, The influence of surface chemistry on the development of minor species profiles in the premixed boundary layer combustion of an $\mathrm{H}_{2}$ /air mixture, Combust. Sci. Technol. 79 (1991) 247.

[10] P. Markatou, L.D. Pfefferle, M.D. Smooke, A computational study of methane-air combustion over heated catalytic and non-catalytic surfaces, Combust. Flame 93 (1993) 185.

[11] S. Buser, P. Benz, A. Schlegel, H. Bockhorn, Measurement of $\mathrm{OH}$ by LIF and temperatures by holographic interferometry in catalytically supported combustion of hydrogen: comparison of measurement and numerical simulation, Ber. Bunsenges. Phys. Chem. 97/12 (1993) 1719.

[12] B.K. Harrison, W.R. Ernst, Catalytic combustion in cylindrical channels: a homogeneous-heterogeneous model, Combust. Sci. Technol. 19 (1978) 31.

[13] C. Bruno, P.M. Walsh, D. Santavicca, F.V. Bracco, Catalytic combustion of propane/air mixtures on platinum, Combust. Sci. Technol. 31 (1983) 43.

[14] U. Dogwiler, P. Benz, J. Mantzaras, Two-dimensional modelling for catalytically stabilized combustion of a lean methane-air mixture with elementary homogeneous and heterogeneous chemical reactions, Combust. Flame 116 (1999) 243.

[15] U. Dogwiler, J. Mantzaras, P.Benz, B. Kaeppeli, R. Bombach, A. Arnold, Homogeneous ignition of methane/air mixtures over platinum: comparison of measurements and detailed numerical predictions, in: Proceedings of the 27th International Symposium on Combustion, The Combustion Institute, 1998, p. 2275.

[16] O. Deutschmann, R. Schmidt, F. Behrendt, J. Warnatz, Numerical modeling of catalytic ignition, in: Proceedings of the 26th International Symposium on Combustion, The Combustion Institute, 1996, p. 1747.

[17] J. Mantzaras, Geometrical properties of turbulent premixed engine flames: comparison of measurements and detailed numerical predictions, Combust. Sci. Technol. 86 (1992) 135.

[18] S.J. Kline, N.H. Afgan, N.H. (Eds.), Near-Wall Turbulence, Hemisphere, New York, 1988.

[19] T.C. Bond, R.A. Noguchi, C.P. Chou, R.K. Mongia, J.Y. Chen, R.W. Dibble, Catalytic oxidation of natural gas over supported platinum: flow reactor experiments and detailed numerical modeling, in: Proceedings of the 26th International Symposium on Combustion, The Combustion Institute, 1996, p. 1771.
[20] H.C. Chen, V.C. Patel, Near-wall turbulence models for complex flows including separation, AIAA J. 26/6 (1988) 641.

[21] W. Rodi, Experience with two-layer models combining the $k-\varepsilon$ model with a one-equation model near the wall, AIAA-91-0216, 29th Aerospace Science Meeting, NV, USA, 1991.

[22] D.C. Wilcox, Turbulence Model for CFD, DCW Industries Inc., La Cañada, CA, USA, 1993.

[23] B.E. Launder, D.B. Spalding, The numerical computation of turbulent flows, Comput. Meth. Appl. Mech. Energy 3 (1974) 269.

[24] V.C. Patel, W. Rodi, G. Scheuerer, Turbulence models for near-wall and low Reynolds number flows: a review, AIAA J. 23 (9) (1984) 1308.

[25] H. Bockhorn, Modeling of turbulent diffusion flames with detailed chemistry, in: C.M. Brauner, C. Schmidt-Laine (Eds.), Mathematical Modeling in Combustion and Related Topics, Martinus Nijhoff, Dordrecht, 1988, p. 411.

[26] H. Bochkorn, Finite chemical reaction rate and local equilibrium effects in turbulent hydrogen-air diffusion flames, in: Proceedings of the 22nd International Symposium on Combustion, The Combustion Institute, 1988, p. 655.

[27] R.K. Cheng, R.G. Bill, Jr., F. Robben, Experimental study of combustion in a turbulent boundary layer, in: Proceedings of the 18th International Symposium on Combustion, The Combustion Institute, 1981, p. 1021.

[28] J. Wardana, T. Ueda, M. Mizomoto, Structure of turbulent two-dimensional channel flow with strongly heated wall, Exp. Fluids 13 (1992) 17.

[29] D.E. Rumelhart, J.L. McClelland, Parallel Distributed Processing: Explorations in the Microstructure of Cognition, MIT Press, Cambridge, MA, 1988.

[30] J. Warnatz, U. Maas, Technische Verbrennung, Springer, Berlin, 1993, p. 101.

[31] J. Warnatz, M.D. Allendorf, R.J. Kee, M.E. Coltrin, A model of elementary chemistry and fluid mechanics in the combustion of hydrogen on platinum surfaces, Combust. Flame 96 (1994) 393.

[32] S.K. Srivatsa, Cham computer code 201, CORA2 - a computer code for axisymmetrical combustion chambers, CHAM TR 201/1, 1977.

[33] R.J. Kee, F.M. Rupley, J.A. Miller, The Chemkin thermodynamic database, Sandia National Laboratories Report No. SAND87-8215B UC-4, July 1996, reprint.

[34] M.E. Coltrin, R.J. Kee, F.M. Rupley, Surface chemkin: a Fortran package for analyzing heterogeneous chemical kinetics at a solid surface-gas phase interface, Sandia National Laboratories Report No. SAND90-8003C UC-706, July 1996, reprint.

[35] J. Mantzaras, P. Benz, An asymptotic and numerical investigation of homogeneous ignition in catalytically stabilized channel flow combustion, Combust. Flame 119 (1999) 455.

[36] C.K. Law, G.I. Sivashinsky, Catalytic extension of extinction limits of stretched premixed flames, Combust. Sci. Technol. 29 (1982) 277. 